\title{
Brewster effect when approaching exceptional points of degeneracy Epsilon-near-zero behavior
}

\section{Popov, Vladislav; Tretyakov, Sergei; Novitsky, Andrey}

\section{Published in:}

Physical Review B

Link to article, DOI:

10.1103/PhysRevB.99.045146

Publication date:

2019

Document Version

Publisher's PDF, also known as Version of record

Link back to DTU Orbit

Citation $(A P A)$ :

Popov, V., Tretyakov, S., \& Novitsky, A. (2019). Brewster effect when approaching exceptional points of degeneracy: Epsilon-near-zero behavior. Physical Review B, 99(4), [045146].

https://doi.org/10.1103/PhysRevB.99.045146

\section{General rights}

Copyright and moral rights for the publications made accessible in the public portal are retained by the authors and/or other copyright owners and it is a condition of accessing publications that users recognise and abide by the legal requirements associated with these rights.

- Users may download and print one copy of any publication from the public portal for the purpose of private study or research.

- You may not further distribute the material or use it for any profit-making activity or commercial gain

- You may freely distribute the URL identifying the publication in the public portal

If you believe that this document breaches copyright please contact us providing details, and we will remove access to the work immediately and investigate your claim. 


\title{
Brewster effect when approaching exceptional points of degeneracy: Epsilon-near-zero behavior
}

\author{
Vladislav Popov* \\ SONDRA, CentraleSupélec, Université Paris-Saclay, F-91190 Gif-sur-Yvette, France \\ Sergei Tretyakov ${ }^{\dagger}$ \\ Department of Electronics and Nanoengineering, Aalto University, P.O. Box 15500, FI-00076 Aalto, Finland \\ Andrey Novitsky \\ DTU Fotonik, Technical University of Denmark, Ørsteds Plads 343, DK-2800 Kongens Lyngby, Denmark \\ and Department of Theoretical Physics and Astrophysics, Belarusian State University, Nezavisimosti Avenue 4, 220030 Minsk, Belarus
}

(Received 18 August 2018; revised manuscript received 10 January 2019; published 28 January 2019)

\begin{abstract}
We reveal that the phenomenon of full plane-wave transmission without phase accumulation commonly associated with epsilon-near-zero (ENZ) materials does not require vanishing of permittivity. We theoretically connect the phenomenon with the condition of the Brewster effect satisfied at the edges of stop bands (at so-called exceptional points of degeneracy) and show that full transmission without phase accumulation can be observed in various one-dimensional periodic structures. Particularly, exploiting the manifold of exceptional points of degeneracy in one-dimensional all-dielectric periodic lattices, we demonstrate that these structures not only offer a lossless and extremely simple, complementary metal-oxide semiconductor compatible alternative for some applications of ENZ media but exhibit new properties of all-angle full transmission with zero phase delay. The main results of the study can be readily transferred to acoustic and matter waves.
\end{abstract}

DOI: 10.1103/PhysRevB.99.045146

\section{INTRODUCTION}

Metamaterials promise amazing possibilities in manipulation of electromagnetic fields, which are not available with natural materials (see, e.g., [1-5]). Extreme properties and unique effects leading to novel functionalities require artificial materials with extreme and singular values of material parameters. Recently, much attention has been paid to the exotic properties of materials with permittivity $\varepsilon$ near zero. Both natural substances and metamaterials possessing $\varepsilon \approx 0$ are called epsilon-near-zero (ENZ) media. From the physical point of view, this special value of permittivity corresponds to the topological transition between metals and dielectrics, as can be illustrated by tuning the parameters of a hyperbolic metamaterial, whose wave dispersion changes from hyperbolic to elliptic type [6,7] at the ENZ point. From the applications point of view, properties of ENZ media can be exploited for energy tunneling through subwavelength channels [8,9], improvement of antenna directivity [10], phase matching due to zero phase advance in ENZ media [11], enhancement of nonlinear effects [12,13], and more [14-16].

Conditions for $\varepsilon \approx 0$ have been the subject of scrutiny for more than a decade [15,17]. Unfortunately, natural ENZ materials are rather lossy, and this may suppress or even ruin their useful properties [18]. To circumvent this difficulty one may exploit the accidental degeneracy near the $\Gamma$ point in all-dielectric photonic crystals, where the zero-refractive-

\footnotetext{
*uladzislau.papou@centralesupelec.fr

†sergei.tretyakov@aalto.fi

‡anov@fotonik.dtu.dk
}

index properties are available $[19,20]$. On the other hand, superlattices constructed of positive and negative index photonic crystals can also be used [21,22]. However, it is important to keep in mind that all artificial ENZ materials are complex two-dimensional (2D) or three-dimensional (3D) lattices that can be characterized by effective permittivity only under the homogenization condition (period is much smaller than the operating wavelength).

In this study, we show that a physical mechanism behind key properties of ENZ media does not require vanishing of permittivity. We theoretically connect the effect of complete wave tunneling without phase accumulation to the condition of the Brewster effect satisfied when approaching exceptional points of degeneracy (EPDs). This theory opens up a possibility to exploit the manifold of EPDs in all-dielectric periodic lattices in order to emulate properties of ENZ media. Particularly, we demonstrate that one-dimensional (1D) periodic structures can be designed to exhibit ENZ behavior. We validate the theory by demonstrating the complete wave tunneling without phase advance through simple layered structures composed of conventional dielectrics. We also show that there is no phase accumulation throughout the whole thickness of the structure.

\section{MAIN IDEA AND ENZ PROPERTIES OF 1D PERIODIC STRUCTURES}

To explain the analogy between the existence of exceptional points of degeneracy, the Brewster effect, and the properties of ENZ media, let us first consider the well-known analytical solution for the plane wave transmission through an isotropic slab of thickness $d_{s}$, permittivity $\varepsilon_{s}$, and permeability 


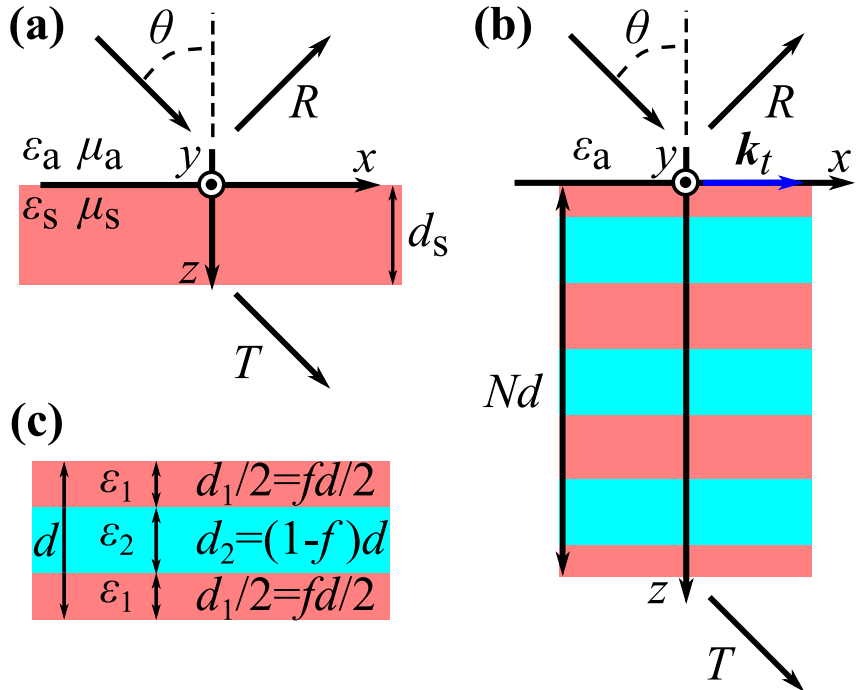

FIG. 1. (a) Reflection and transmission through an isotropic slab and (b) 1D photonic crystal composed of alternating layers of isotropic dielectrics. (c) Three-layer (having an inversion center) unit cells of a 1D photonic crystal.

$\mu_{s}$, as illustrated in Fig. 1(a). The transmission coefficient for an obliquely incident plane wave of arbitrary polarization can be written as [10]

$$
T=\left[\cos \left(k_{z s} d_{s}\right)-i \frac{Z_{s}^{2}+Z_{a}^{2}}{2 Z_{s} Z_{a}} \sin \left(k_{z s} d_{s}\right)\right]^{-1} .
$$

Here, $k_{z s}=\sqrt{\varepsilon_{s} \mu_{s} k_{0}^{2}-k_{t}^{2}}$ is the normal component of the wave vector inside the slab, $k_{0}=\omega / c$ is the vacuum wave number ( $\omega$ is the frequency, $c$ is the speed of light in vacuum), $k_{t}$ represents the tangential component of the wave vector, and $Z_{s}$ and $Z_{a}$ are the wave impedances inside and outside the slab, respectively. The impedances are defined as the ratios of the tangential to the slab plane wave field components. Assuming the absence of losses, the reflectivity $1-|T|^{2}$ vanishes provided $Z_{s}=Z_{a}$. Except for the trivial case of the same materials of the slab and the ambient medium, the equal wave impedances are realized under conditions of Brewster's law. In this case the transmission coefficient $T=e^{i k_{2 s} d_{s}}$ describes the phase shift of the fully transmitted wave. When the full transmission is complemented by zero phase shift $k_{z s} d_{s}=0$, a unique phenomenon of complete wave tunneling without phase accumulation is realized. Originally, it was associated with the ENZ/mu-near-zero (MNZ)/epsilonmu-near-zero (EMNZ) materials.

It is noteworthy that a Fabry-Pérot resonance occurring in any dielectric slab under the conditions $k_{z s} d_{s}=2 \pi m(\mathrm{~m}=$ $1,2, \ldots)$ is also characterized by full transmission and zero phase shift. However, the Brewster effect is fundamentally different from the Fabry-Pérot resonance. It exists at any thickness of the slab $d_{s}$. Moreover, a plane wave does not accumulate phase when it propagates across the slab under conditions $Z_{s}=Z_{a}$ and $k_{z s}=0$. On the output the phase accumulation is zero but not a multiple of $2 \pi$.

Condition $k_{z s}=0$ defines the critical angle $\theta_{c}$ of the total internal reflection (TIR). Here, we utilize only the critical angle condition $k_{z s}=0$, while the phenomenon of TIR itself (a)

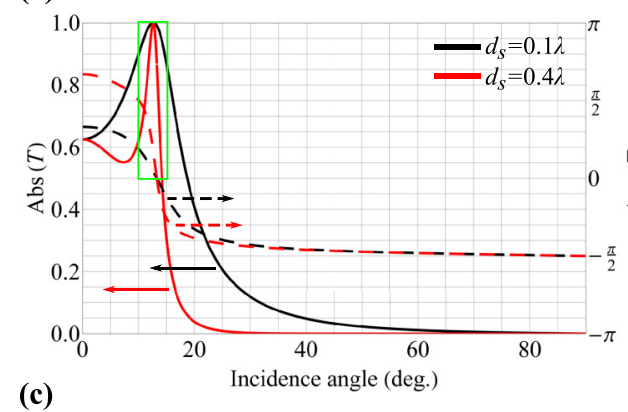

(c)

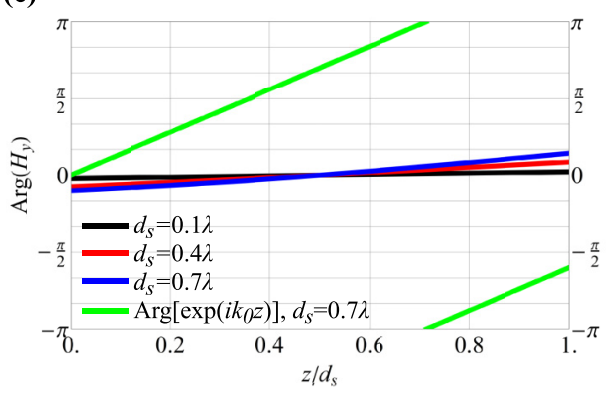

(b)

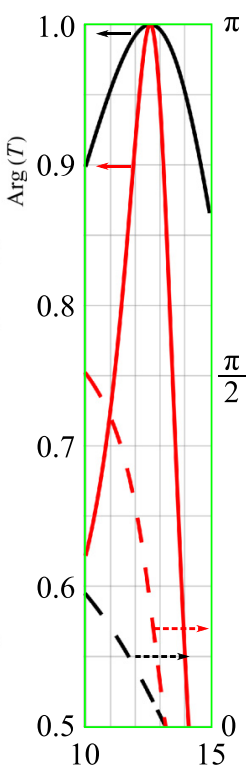

FIG. 2. (a) Transmission coefficient $T$ through a dielectric slab for TM polarization versus incidence angle. (b) Zoom of the boxed area in (a). (c) The phase distribution inside the slab under the critical angle incidence. The green line describes the phase evolution of a plane wave propagating through vacuum within the distance $d_{s}$. In all panels $\lambda$ represents the vacuum wavelength. Parameters are $\varepsilon_{a}=20$, $\mu_{a}=1, \varepsilon_{s}=1$, and $\varepsilon_{s}=1$. The Brewster angle and the critical angle of TIR equal $12.6^{\circ}$ and $12.9^{\circ}$, respectively.

is not observed for the considered slabs, being available only for very thick layers. Since we need the two conditions $Z_{s}=$ $Z_{a}$ and $k_{z s}=0$ to be satisfied simultaneously for full transmission without phase accumulation, the Brewster angle and the critical angle of total internal reflection should be close. Indeed, as illustrated by Fig. 2, the TM-polarized wave is fully transmitted with no phase accumulation for conventional dielectric materials $\left(\varepsilon_{a}=20\right.$ and $\left.\varepsilon_{s}=1\right)$. Even though the Brewster angle $\left(\theta_{B}=12.6^{\circ}\right)$ and the critical angle of TIR $\left(\theta_{c}=12.9^{\circ}\right)$ do not coincide exactly, the system is highly transparent at $\theta=\theta_{c}$ [see Fig. 2(b)]. Similar to an ENZ case the phase accumulation across the slab is insignificant, unlike the unbounded plane wave propagation in vacuum, as shown in Fig. 2(c). Interestingly, the investigated phenomenon does not suffer from frequency dispersion because the critical angle of TIR and Brewster's angle depend only on the practically dispersionless material parameters of the dielectrics and not on the frequency. It is demonstrated in Fig. 2(b) with the help of two curves corresponding to different thicknesses $d_{s}$ of the slab. Thus, we have obtained surprising results demonstrating that the permittivity should not necessarily vanish to achieve high transmission without phase accumulation. Instead, under specific conditions, conventional dielectrics can be exploited.

Generally speaking, the critical angle of TIR corresponds to an EPD since the condition $k_{z s}=-k_{z s}=0$ means that the propagation constants of the oppositely directed waves coincide (degenerate), while the eigenvectors and hence eigenwaves' impedances are indistinguishable. Thus, the phenomenon of full transmission without phase accumulation can be found for many different structures. Indeed, let us consider 
a simple (for analytical derivations) example of a 1D periodic structure which can be described by an ABCD matrix [23] (or, in other words, transfer matrix) and has a symmetric unit cell (in this case $A=D$ [23]) of geometric size $d$. The asymmetric case $(A \neq D)$ is discussed in Appendix A. Bloch waves characterized by a wave number $q$ and wave impedance $Z_{B}$ propagate through the periodic structure. Keeping in mind the unimodularity of the ABCD matrix $\left(A^{2}-B C=1\right)$ for the nondissipative structure, one can write the Bloch wave number $q= \pm d^{-1} \cos ^{-1}(A)$ and wave impedance $Z_{B}= \pm \sqrt{C / B}$, solving the eigenvalue and eigenvector problem for the $\mathrm{ABCD}$ matrix [23]. Here, plus and minus signs correspond to forward and backward Bloch waves. They cannot be distinguished at the edges between stop bands and passbands (i.e., when $q=0$ and $\pi / d$ ), where the wave impedance diverges or vanishes by means of $B=0$ or $C=0$. Thus, the edges of stop bands can always be treated as EPDs.

The transmission coefficient through an $N$-cell 1D periodic structure is given by the following formula:

$$
T=\left[\cos (q N d)-i \frac{\frac{C}{B}+Z_{a}^{2}}{2 Z_{a}} \sqrt{\frac{B}{C}} \sin (q N d)\right]^{-1},
$$

which is basically the same as Eq. (1), but the wave impedance of the Bloch wave $Z_{B}$, Bloch wave number $q$, and $N d$ are substituted for, respectively, $Z_{s}, k_{s z}$, and $d_{s}$. At Brewster angle incidence $Z_{a}=Z_{B}$ and the transmission coefficient Eq. (2) is reduced to the phase factor $T=\exp [i q N d]$. On the other hand, when approaching EPDs, we find [keeping in mind that $\sin (q d)=\sqrt{-B C}$ ] that the transmission coefficient approaches the following limits:

$$
T \rightarrow\left\{\begin{array}{cc}
{\left[1-N C /\left(2 Z_{a}\right)\right]^{-1},} & Z_{B} \rightarrow \infty, \\
\left(1-N B Z_{a} / 2\right)^{-1}, & Z_{B} \rightarrow 0 .
\end{array}\right.
$$

Although none of these limits equal unity, the transmission coefficient can approach unity arbitrarily closely when the Brewster condition $Z_{B}=Z_{a}$ is satisfied close to a band gap edge.

As in the case of a homogeneous ENZ slab, the wave does not accumulate phase when propagating over periods of the structure. Again, there are two principal situations corresponding to the incident plane wave exactly matched with (i) the Brewster condition or (ii) a stop band edge. In the first case, the incident wave is impedance matched with the forward Bloch wave, and phases of both magnetic and electric fields vary as $q N d$. In the other situation one has to turn to the ABCD matrix, which at a stop band edge has $A=1$ and either $B=0(C \neq 0)$ or $C=0(B \neq 0)$. The spatial evolution is given by the ABCD matrix acting on the field column $\left[1+R, Z_{a}(1-R)\right]^{T}$ at the input of the structure $(R$ is the reflection coefficient). When $B=0$, the magnetic field does not change throughout the structure and has the phase $\operatorname{Arg}(H)=\operatorname{Arg}(1+R)$. Meanwhile, the phase profile of the electric field is given by the equation $\operatorname{Arg}(E)=\operatorname{Arg}(N C[1+$ $R]+Z_{a}[1-R]$ ). If $C$ is zero and $B$ is not, the electric field is constant with the phase $\operatorname{Arg}(E)=\operatorname{Arg}(1-R)$, and the phase of the magnetic field changes as $\operatorname{Arg}(H)=\operatorname{Arg}\left(N B Z_{a}[1-\right.$ $R]+1+R)$. These situations become indistinguishable when the Brewster condition occurs at a stop band edge.

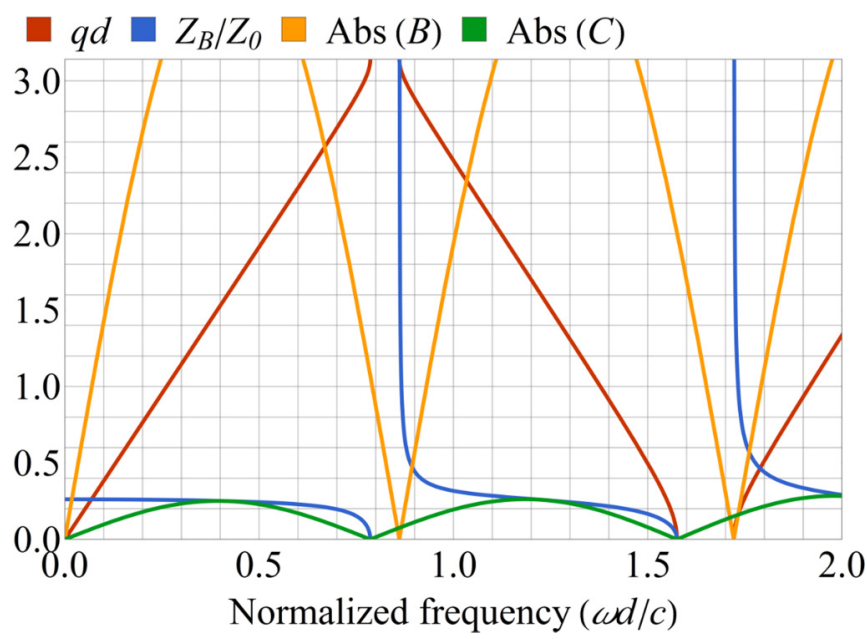

FIG. 3. Frequency dependence of Bloch wave number $q$, wave impedance $Z_{B} / Z_{0}=\sqrt{C / B} / Z_{0}$ (normalized to the free-space impedance $Z_{0}$ ), and functions $B$ and $C$ corresponding to the periodic multilayer structure illustrated by Figs. 1(b) and 1(c). Parameters are $\theta=0$ (wave impedance of a normally incident plane wave is $Z_{0}$ ), $\varepsilon_{1}=2, \varepsilon_{2}=16$, and $f=0.1$.

To conclude this section, a discrete analog of the wave propagation phenomenon in homogeneous ENZ media can be achieved in periodic structures. To that end one needs to appropriately design a unit cell to get a required $\mathrm{ABCD}$ matrix, e.g., a design of microwave networks is discussed in Ref. [23].

\section{APPLICATION OF A 1D PHOTONIC CRYSTAL}

As a concrete example we consider a 1D photonic crystal represented by a periodic multilayer composed of three-layer unit cells with an inversion center [24], as shown in Figs. 1(b) and $1(\mathrm{c})$. The components of the ABCD matrix corresponding to this structure can be found analytically as presented in Appendix B. EPDs are well studied in 1D photonic crystals (see, e.g., Refs. [25-27]) and correspond to edges of stop bands $(A= \pm 1)$ where wave impedance diverges or vanishes (at zeros of functions $B$ and $C$, respectively). Owing to the axial symmetry stop band edges can be treated as surfaces of degeneracy. Figure 3 illustrates an example of the frequency dependence of the wave number and wave impedance of a Bloch wave propagating in a periodic multilayer.

\section{A. Wide-angle full transmission without phase accumulation}

Complete wave tunneling without phase accumulation in 1D photonic crystals is available for any incidence angle $\theta$ and any polarization if the boundaries of the stop bands are almost flat. The required band structure can be achieved for alternating low- and high-permittivity dielectric layers with a small filling fraction $f=d_{1} / d$ of the low-permittivity dielectric. Then the stop bands become narrower, while their edges are almost flat for both TE [Fig. 4(a)] and TM polarizations [Fig. 4(b)]. The full transmission $|T|=1$ independent of the total thickness $N d$ (Brewster's resonance) is realized at $Z_{B}=Z_{a}$. The values of the incidence angles and frequencies 
(a)

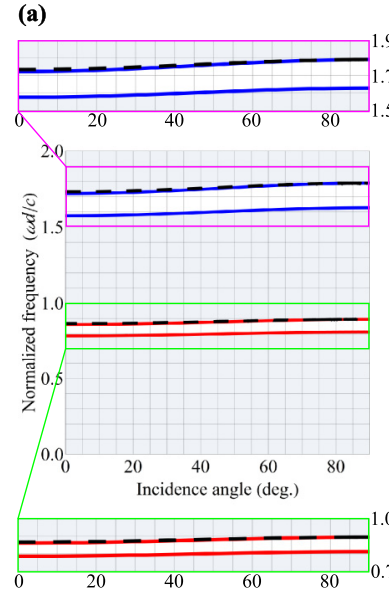

(b)

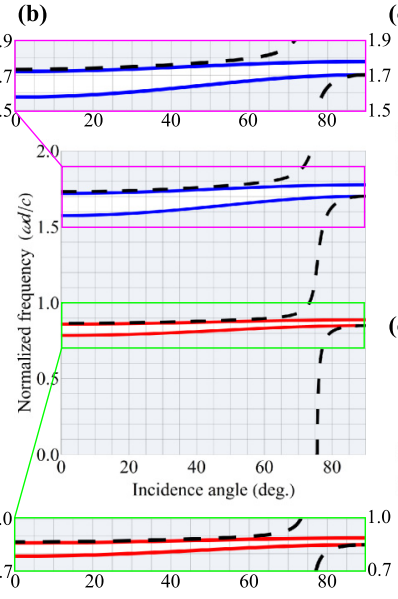

(c)

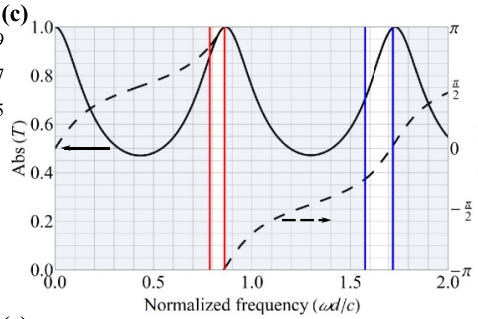

(e)

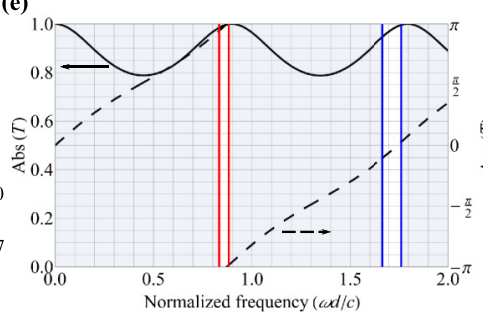

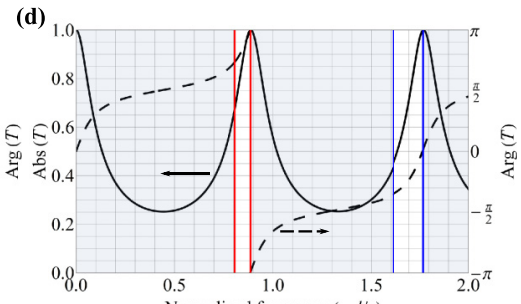

(f)

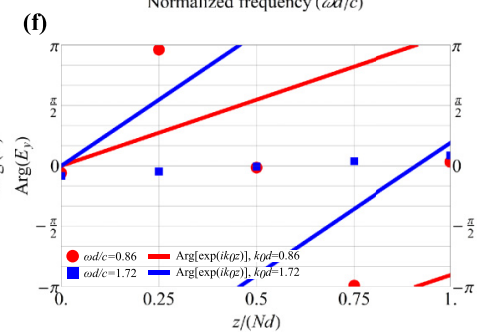

FIG. 4. Band gap structure for (a) TE and (b) TM polarizations of the 1D photonic crystal depicted in Fig. 1(b). The black dashed curves correspond to Brewster's law. (c)-(e) Absolute value and phase of the transmission coefficient through a single unit cell of the photonic crystal versus the normalized frequency: (c) normal incidence, (d) TE polarization, $\theta=60^{\circ}$, and (e) TM polarization, $\theta=60^{\circ}$. In all panels the shaded regions represent propagation bands, and red and blue curves and lines represent the edges of stop bands where $q=\pi / d$ and $q=0$, respectively. (f) Spatial evolution of the electric field phase over periods of the four-cell 1D photonic crystal (circles and squares) compared to the phase accumulation of a plane wave propagating through a vacuum region of equivalent thickness (solid lines). Parameters are $\varepsilon_{1}=2$, $\varepsilon_{2}=16$, and $f=0.1$.

at which Brewster's law in periodic multilayers is satisfied are depicted as dashed lines in Figs. 4(a) and 4(b). It should be noticed that Bloch impedance $Z_{B}$ is frequency dependent, which gives rise to frequency dispersion. For small filling fractions $f$, the curves $Z_{B}=Z_{a}$ shift to the top edges of the stop bands, thus providing both full transmission and zero phase accumulation.

The transmission coefficient through a single unit cell $(N=1)$ as a function of the normalized frequency $k_{0} d=$ $\omega d / c$ is demonstrated in Figs. 4(c)-4(e). All maxima in Figs. 4(c)-4(e) correspond to Brewster's resonances, which are independent of the total thickness $N d$. There are no FabryPérot resonances at the band gap edges $q=0$ and $q=\pi / d$ in Figs. 4(c)-4(e) since $Z_{B}$ goes either to zero or infinity, while the term $\left(Z_{B}^{2}+Z_{a}^{2}\right) /\left(2 Z_{B} Z_{a}\right) \sin (q N d)$ takes a nonzero value and therefore $|T|<1$ according to Eq. (3). On the contrary, when the Brewster condition $Z_{B}=Z_{a}$ is satisfied next to stop band edges, the transmission coefficient can approach unity arbitrarily closely when a sufficiently small $f$ is chosen (of course, $f$ cannot be exactly zero as there would be no band gap). Except for the case of normal incidence $\theta=0$, the multilayer structure is polarization sensitive. When the incidence angle of the TE (TM) wave increases, the stop bands get wider (narrower) [see Figs. 4(a) and 4(b)], while the widths of the transmission resonances shown in Figs. 4(c)-4(e) decrease (increase). Transmission coefficients for the waves at the top edges of stop bands $q=\pi / d$ and $q=0$ possess the phases $\pi$ and 0 , respectively, as shown by dashed curves in Figs. 4(c)-4(e).

Figure 4(f) demonstrates the phase of the electric field at the points multiple to the period of the four-cell multilayer. At the top edge of the first stop band $q=\pi / d$ the phases are close for even and odd periods, and there is no phase accumulation at discrete points $z_{m}=2 m d$ and $z_{m}=(2 m+$ $1) d$ ( $m$ is an integer number). At the top edge of the second stop band $q=0$ the phases are nearly equal after every period of the multilayer; that is, there is no phase accumulation at discrete points $z_{m}=m d$. To compare, plane waves propagating in an unbounded vacuum of the same thickness experience significant phase accumulation, as shown by the solid lines in Fig. 4(f). We should stress that the phase within each unit cell changes significantly, although the phase accumulation between the periodically arranged points is almost absent.

The polarization-insensitive illusion effect represents an impressive demonstration of the discussed phenomenon. An ideal device making an illusion is invisible; therefore, its realization using a 1D photonic crystal, which completely tunnels the wave without a phase advance for a vast range of incident angles, is natural. In Fig. 5, a source is placed below the photonic crystal and radiates at the frequencies specified by the top edge of the stop band. Then an observer sees the source at a shorter distance, the displacement towards the observer being equal to the thickness of the photonic crystal slab. Such an illusion is demonstrated in Fig. 5 for magnetic and electric line sources, i.e., for TE and TM polarizations. It is worth noticing that an illusion phenomenon was reported in Refs. [28-31] on the basis of transformation optics.

\section{B. ENZ-like spatial frequency filtering}

A thick epsilon-near-zero slab acts as an effective filter of spatial frequencies transmitting only normally incident waves. Although in the case of 1D photonic crystals it is difficult to get such a functionality for the normally incident wave, sharp transmission resonances are very accessible for grazing incident waves when the wave vector of the incident wave is almost parallel to the photonic crystal interfaces. To that end, we study a multilayer composed by alternating low- and high-permittivity dielectric layers, keeping the filling fraction $f=d_{1} / d$ of the low-permittivity dielectric high. Figures 6(a) 


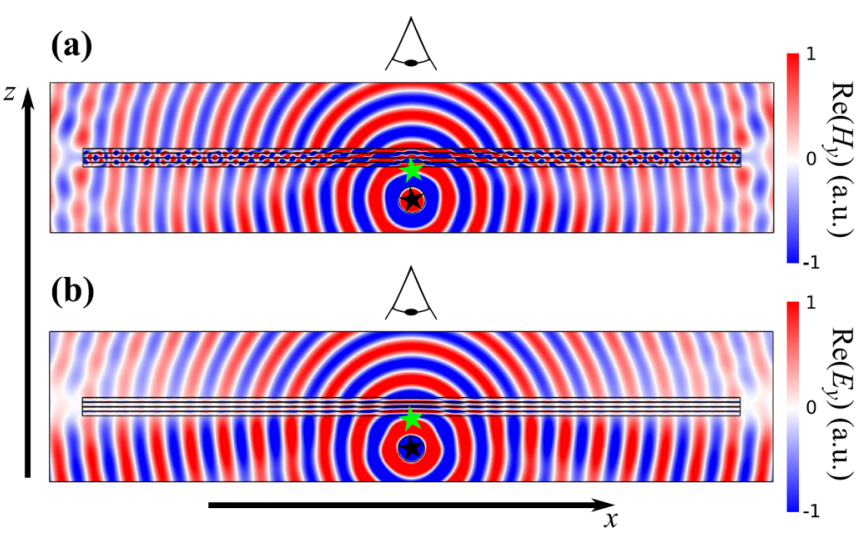

FIG. 5. (a) Distribution of the magnetic field when a magnetic line source illuminates a 1D photonic crystal. (b) Distribution of the electric field when an electric line source illuminates the photonic crystal. Normalized frequency is $k_{0} d=0.86$, the distance to the photonic crystal is $\lambda$, the $1 \mathrm{D}$ photonic crystal has $N=4$ three-layer unit cells, and the length is $20 \lambda$. The eye represents an observer who sees an illusion (top star) of the source (bottom star).

and 6(c) depict the band gap structure for TM and TE polarizations. The Brewster condition is met at the black dashed curves. Blue and red curves correspond to the edges of stop bands $q=0$ and $q=\pi / d$, respectively. By magnifying the green boxed areas we clearly see from Figs. 6(b) and 6(d) that the Brewster effect occurs at a stop band edge for both polarizations (but at different frequencies) when the incidence angle $\theta$ approaches $90^{\circ}$. In Figs. 6(e) and 6(f) we plot the angular dependence of the transmission coefficient at the corresponding frequencies for photonic crystals composed of
10 (solid curve) and 30 (dashed curve) unit cells. Since the whole range of incidence angles apart from the vicinity of $90^{\circ}$ corresponds to a stop band, highly selective transmission is observed. The transmission resonances do not depend on the total thickness $N d$ of the multilayer; that is, they are Brewster's resonances. When the Brewster condition is satisfied at a stop band edge, the phase of the transmission coefficient vanishes. It can be seen from the frequency dependence of the transmission coefficient around the stop band edge frequency (the incidence angle is $89.9^{\circ}$ ), shown in Figs. 6(g) and 6(h), where one can actually recognize both the Brewster and Fabry-Pérot resonances. The Brewster resonances appear right at the stop band edge.

Full power transmission also occurs at Fabry-Pérot resonances, where $q N d=\pi m$ ( $m$ is an integer); in this case the transmission coefficient given by Eq. (2) is $T=1 / \cos (\pi m)$. The Fabry-Pérot resonances are seen in Figs. 6(g) and 6(h) at frequencies below the Brewster peak at the stop band edge. Interestingly, for even $m$ we have $T=1$, but the phase accumulation is $2 \pi \mathrm{m}$. As noted above, the Brewster effect is independent of $N d$, and there is no phase accumulation in the full transmission regime, which makes it fundamentally different from the Fabry-Pérot resonances.

The absence of phase accumulation over the full length of the photonic crystal with 30 unit cells is demonstrated by Fig. 7. Field phases at the interfaces of unit cells are shown at an incidence angle of $89.9^{\circ}$. In the case of the TM polarization the stop band edge of interest corresponds to $q=\pi / d\left(k_{0} d \approx\right.$ $1.96)$, and there is no phase accumulation between discrete points $z_{m}=2 m d$ and $z_{m}=(2 m+1) d$ ( $m$ is an integer). For the TE polarization the Brewster condition is satisfied at the stop band edge $q=0$ when the frequency is approximately (a)

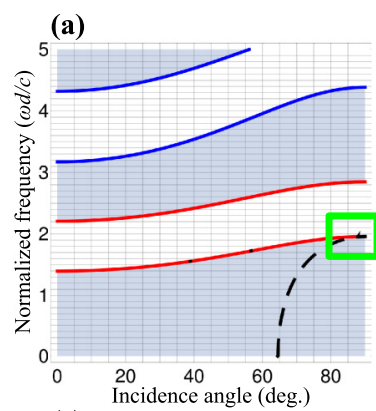

(c)

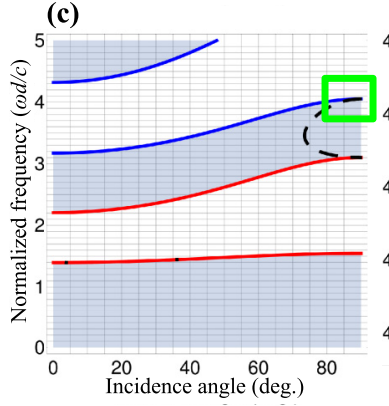

(b)

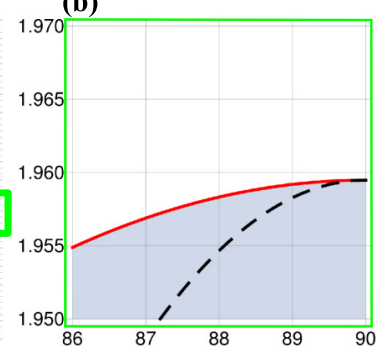

(d)

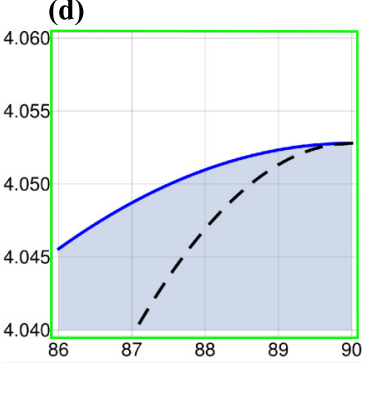

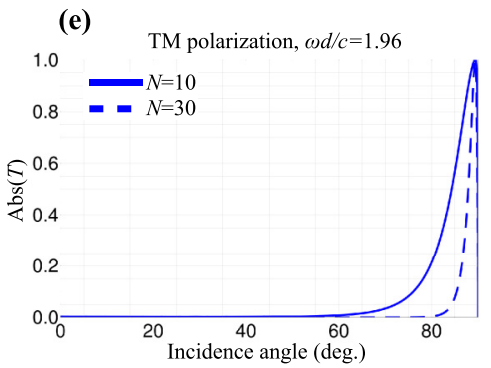
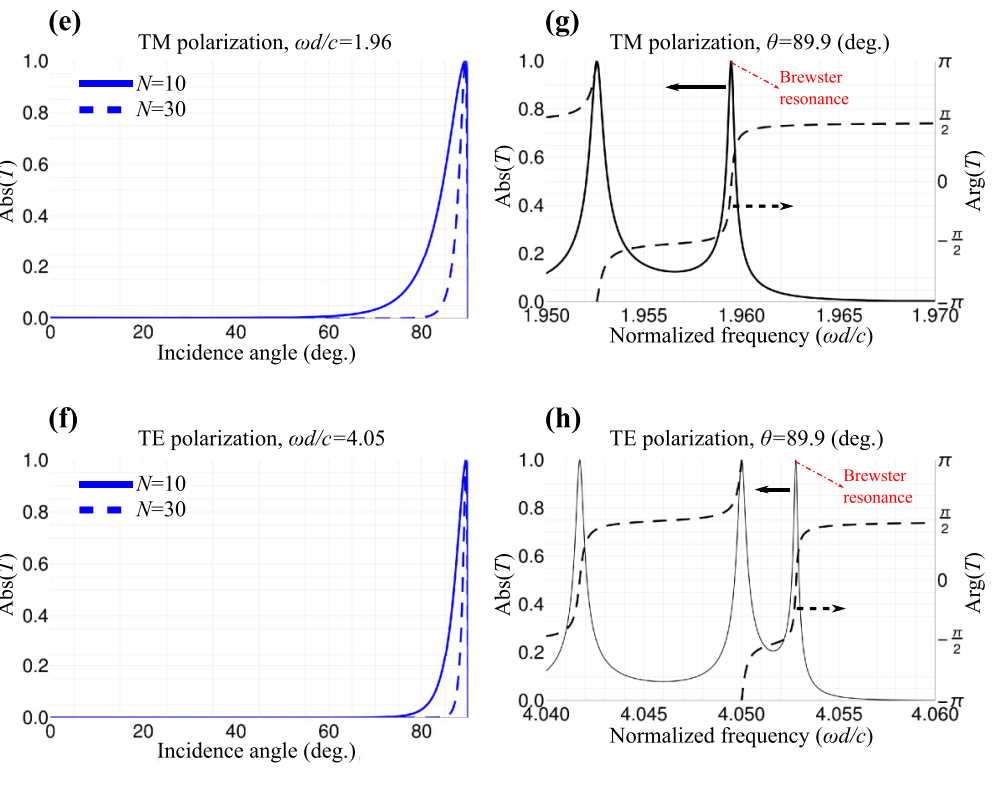

(h)

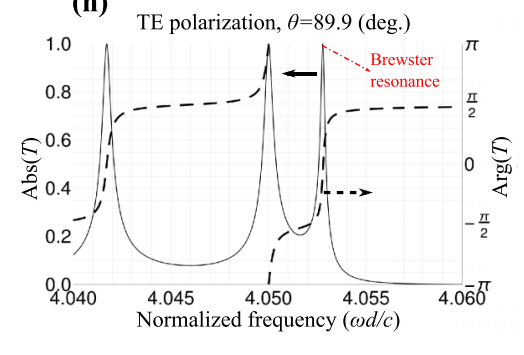

FIG. 6. (a) and (c) Band gap structures of the photonic crystal illustrated in Fig. 1(b): (a) TM polarization and (c) TE polarization. (b) and (d) Zooms of the boxed areas in (a) and (c), respectively. The Brewster condition is satisfied at the black dashed curves. Shaded regions represent propagation bands; red and blue curves represent the edges of stop bands where $q=\pi / d$ and $q=0$, respectively. (e) and (f) Angular dependence of the transmission coefficient through slabs of photonic crystals with 10 and 30 unit cells. (g) and (h) Frequency dependence of the transmission coefficient through the 30 -unit-cell photonic crystal. In all panels the material parameters are $\varepsilon_{1}=2, \varepsilon_{2}=16$, and the volume fraction of the low-index material is $f=0.9$. 


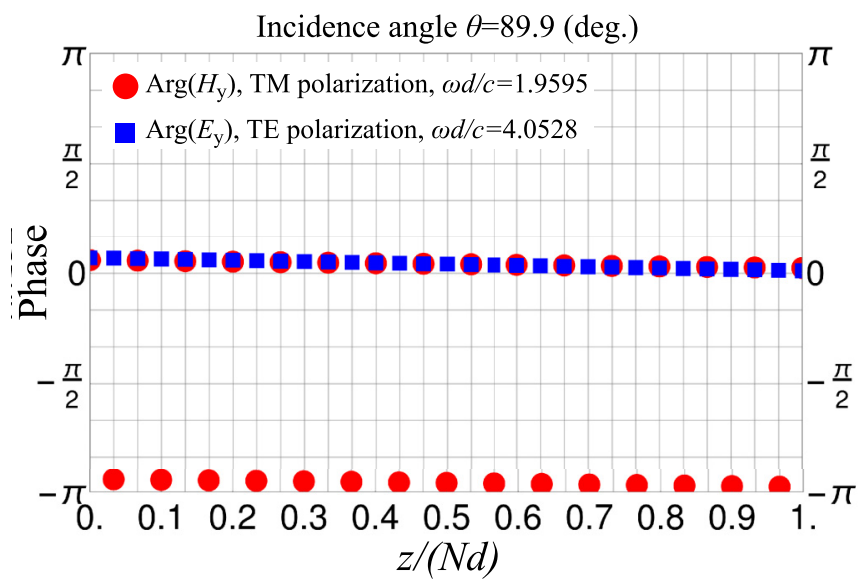

FIG. 7. Distribution of the field phase throughout the photonic crystal with 30 unit cells for cases of TM $\left(k_{0} d=1.9595\right)$ and TE $\left(k_{0} d=4.0528\right)$ polarizations. The points represent the field phase at the interfaces of unit cells. The structure parameters are $\varepsilon_{1}=2$, $\varepsilon_{2}=16$, and $f=0.9$.

$k_{0} d=4.05$, and zero phase accumulation is observed after every period.

To conclude this section let us note that the results represented by Figs. 6 and 7 look nicer than those demonstrated in Sec. III A. This is due to the fact that the wave impedances of incident plane waves diverge for TE polarization and vanish in the case of the $\mathrm{TM}$ one at $90^{\circ}$ incidence. Meanwhile, the wave impedances of Bloch waves behave the same at stop band edges. Thus, the Brewster condition is satisfied exactly at the considered stop band edges.

\section{DISCUSSION AND CONCLUSION}

Realistic losses and random fluctuations of geometrical parameters of the dielectric structures may not affect the overall performance much, in sharp contrast to the ENZ media [18]. Additionally, it is worth noting that we have considered only propagation of monochromatic plane waves; however, an information-carrying signal would consist of a spectrum of such waves. From Figs. 4 and 6 one can get information about the frequency response and, particularly, see that the group delay $\partial \operatorname{Arg}(T) / \partial \omega$ (an important characteristic in signal processing [32]) increases when approaching the frequencies of the top edges of stop bands ( in agreement with the wellknown slow-light phenomenon [33]). This observation brings us to the conclusion that only spectrally narrow signals can propagate without distortion through the considered photonic crystal when showing ENZ properties. More details on the structure presented in Sec. III A can be found in Appendix C.

To summarize, we have identified the physical mechanism behind the properties of ENZ media, namely, the Brewster effect occurring next to exceptional points of degeneracy of an electromagnetic structure. This has allowed us to demonstrate that vanishing of permittivity is not required for achieving full transmission without phase accumulation and can be realized with many different $1 \mathrm{D}$ periodic structures. Particularly, we demonstrated the phenomenon using simple layered structures composed of conventional dielectrics. These structures not only offer a lossless and extremely simple complementary metal-oxide semiconductor compatible alternative for some applications of ENZ media but exhibit new properties of all-angle polarization-insensitive full transmission with zero phase accumulation. Weak sensitivity of the wave tunneling property to variations of electromagnetic and geometrical parameters of the structure makes this system attractive for the realization of such extreme effects as source-displacement illusion. Since exceptional points of degeneracy are ubiquitous, we expect that one can also realize ENZ-like properties with more complex 2D and 3D structures not capable of being homogenized. Finally, we would like to note that the main results of the study can be readily transferred to acoustic and matter waves, pushing forward the development of novel acoustic and electronic devices.

\section{APPENDIX A: ENZ BEHAVIOR IN 1D PERIODIC STRUCTURES WITH AN ASYMMETRIC UNIT CELL}

Electromagnetic wave propagation through onedimensional periodic structures can be described by means of the ABCD matrix approach [23]. In the main text, we discuss conditions of full transmission without phase accumulation for waves penetrating periodic structures with a symmetric unit cell. Here, we focus on the distinct case of an asymmetric unit cell resulting in $A \neq D$. Then, a Bloch wave number $q$ is found to be [23]

$$
q= \pm \frac{1}{d} \cos ^{-1}\left[\frac{A+D}{2}\right]
$$

Meanwhile, wave impedances of the eigenwaves are given by the equation

$$
Z_{B}^{ \pm}=\frac{C}{\frac{A-D}{2} \mp i \sin (q d)} .
$$

The top and bottom sings correspond to waves propagating along and against the $z$ axis, respectively. From formula (A2) it follows that $Z_{B}^{+} \neq-Z_{B}^{-}$in this general case. Furthermore, even in the lossless scenario the wave impedance $Z_{B}^{ \pm}$is complex valued, contrary to the case of symmetric unit cells when the impedance is either real (passband band) or imaginary (stop band). Then, formula (1) for the transmission coefficient takes the following more general form:

$$
T=\left[\cos (q N d)-i \frac{Z_{a}^{2}-Z_{B}^{+} Z_{B}^{-}}{Z_{a}\left(Z_{B}^{+}-Z_{B}^{-}\right)} \sin (q N d)\right]^{-1} .
$$

Let us consider the behavior of the transmission coefficient at a stop band edge. It may seem that the transmission coefficient equals 1 when, e.g., $q=0$. However, when approaching a boundary of a stop band, $\sin (q d) \rightarrow 0$, and accordingly, $Z_{B}^{+} \rightarrow Z_{B}^{-}$. That is, wave impedances at a stop band boundary are not independent, while the expression for the difference $\left(Z_{B}^{+}-Z_{B}^{-}\right)$can be reduced as follows:

$$
\begin{aligned}
Z_{B}^{+}-Z_{B}^{-} & =\frac{C}{\frac{A-D}{2}-i \sin (q d)}-\frac{C}{\frac{A-D}{2}+i \sin (q d)} \\
& =\frac{2 C i \sin (q d)}{\left(\frac{A-D}{2}\right)^{2}+\sin (q d)^{2}} .
\end{aligned}
$$



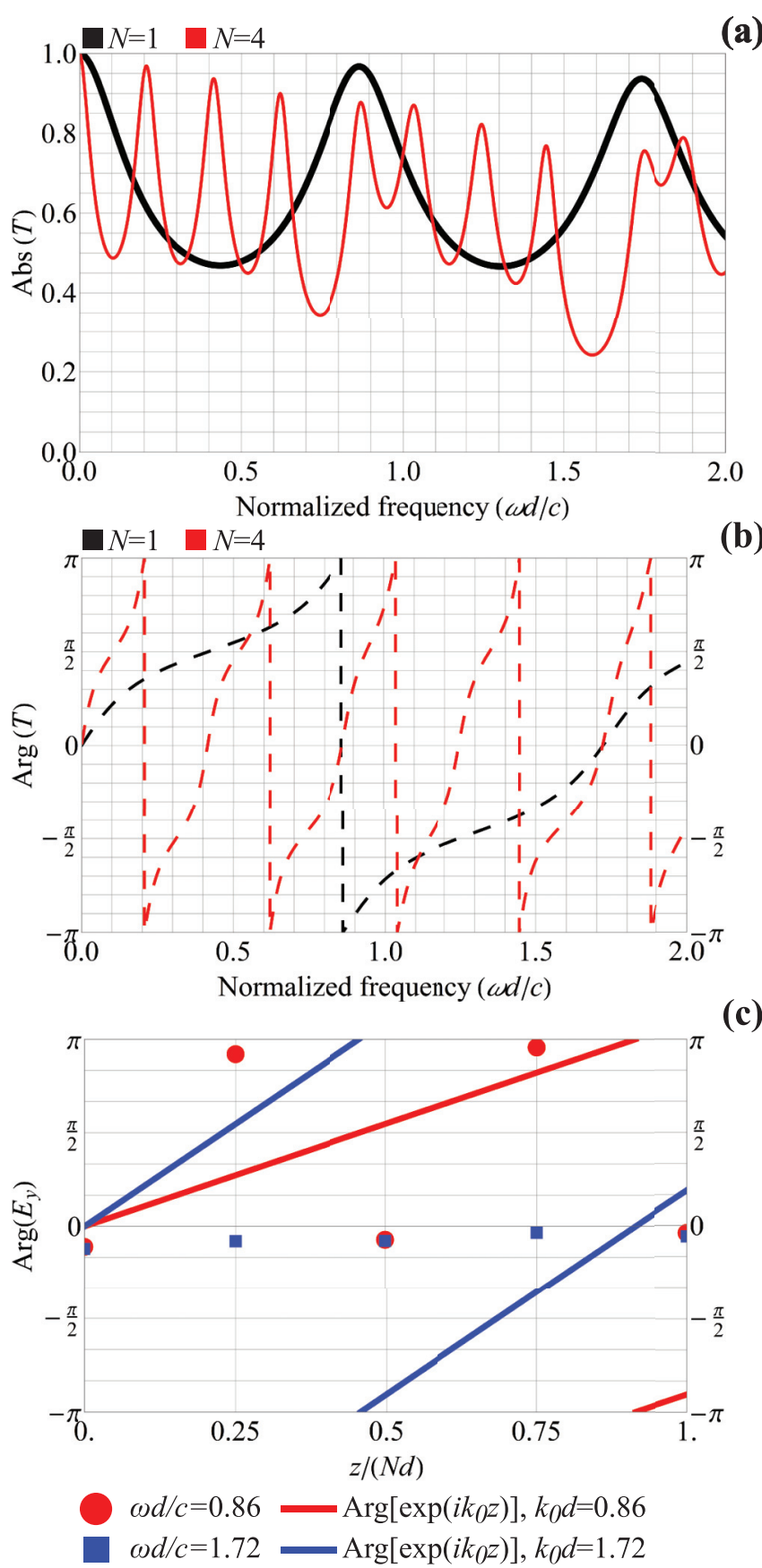

FIG. 8. Frequency dependence of (a) the absolute value and (b) phase of the transmission coefficient through a 1D photonic crystal with $N$ unit cells. (c) Dependence of the field phase on the discrete period's number $n$ of the $1 \mathrm{D}$ photonic crystal with $N=4$ unit cells compared to the phase accumulation of a plane wave propagating through a vacuum region of equivalent thickness. Parameters are normal incidence, $\varepsilon_{a}=1, \varepsilon_{1}=2(1+i 0.01), \varepsilon_{2}=16(1+i 0.01)$, and $f=0.1$. Calculations were conducted under the assumption of $5 \%$ random errors in the thicknesses of each dielectric layer.

As a result, the denominator of the fraction $\left(Z_{a}^{2}-\right.$ $\left.Z_{B}^{+} Z_{B}^{-}\right) /\left[Z_{a}\left(Z_{B}^{+}-Z_{B}^{-}\right)\right]$goes to zero, while the numerator has a nonzero limit. Since the difference $\left(Z_{B}^{+}-Z_{B}^{-}\right)$is proportional to $\sin (q d)$ [when $\sin (q d)$ is close to zero, of course],

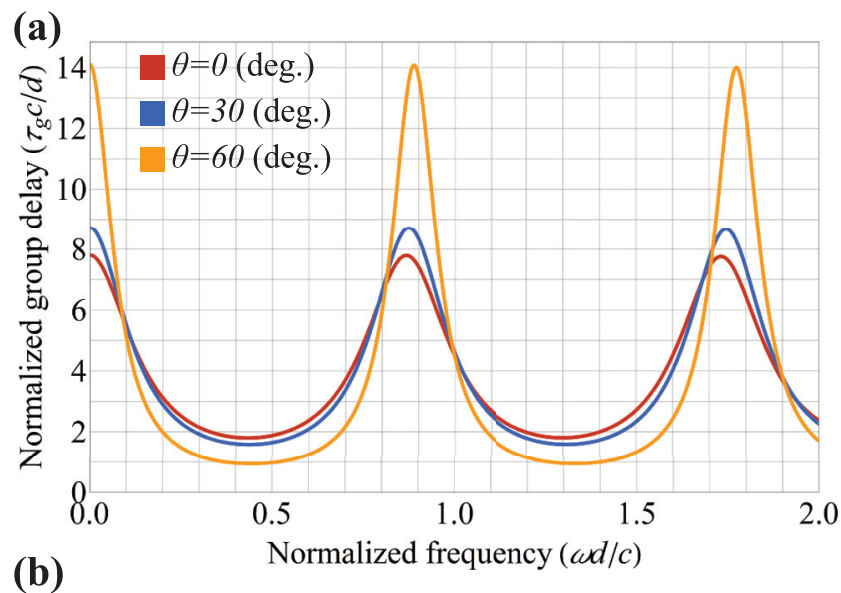

(b)

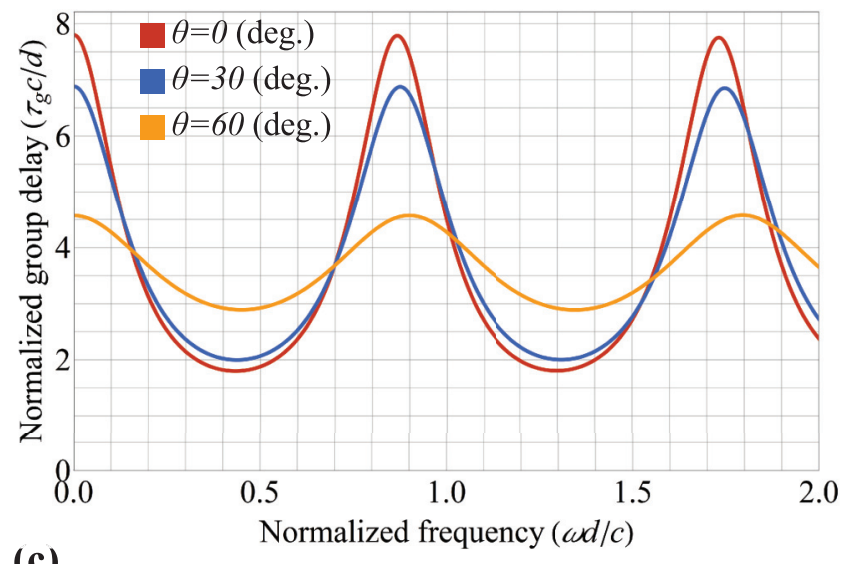

(c)

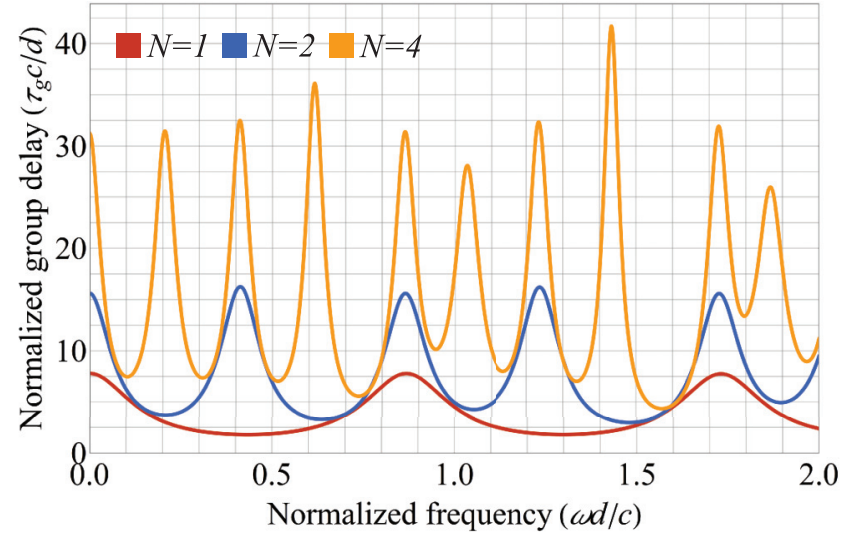

FIG. 9. Frequency dependence of normalized group delay. (a) and (b) Through a single unit cell for different incidence angles in the case of (a) TE and (b) TM polarizations. (c) Through a 1D photonic crystal with different numbers of unit cells $N$ versus normalized frequency in the case of normal incidence. Other parameters are $\varepsilon_{a}=1, \varepsilon_{1}=2, \varepsilon_{2}=16$, and $f=0.1$.

$\sin (q N d) /\left(Z_{B}^{+}-Z_{B}^{-}\right)$also has a nonzero limit proportional to $N$. Eventually, the transmission coefficient does not go to 1 at a stop band boundary. However, if $\sqrt{Z_{B}^{+} Z_{B}^{-}}$were to go to $Z_{a}$ when approaching a stop band boundary, one would observe full transmission without phase accumulation. 


\section{APPENDIX B: ABCD MATRIX OF A 1D PHOTONIC CRYSTAL WITH A SYMMETRIC UNIT CELL}

Spatial evolution of the fields over a period of the 1D photonic crystal considered in Sec. III is described for each polarization by a $2 \times 2 \mathrm{ABCD}$ matrix acting on the field column $(H, E)^{T}$ [where $H$ and $E$ are the tangential components of the magnetic and electric fields, respectively]. The components of the $\mathrm{ABCD}$ matrix are given by the following formulas [24]:

$$
\begin{aligned}
A= & D=\cos \left(k_{z 1} f d\right) \cos \left(k_{z 2}[1-f] d\right) \\
& -\frac{1}{2}\left(\frac{Z_{1}}{Z_{2}}+\frac{Z_{2}}{Z_{1}}\right) \sin \left(k_{z 1} f d\right) \sin \left(k_{z 2}[1-f] d\right), \\
B= & \frac{i}{Z_{1}}\left\{\sin \left(k_{z 1} f d\right) \cos \left(k_{z 2}[1-f] d\right)\right. \\
& +\frac{1}{2}\left[\left(\frac{Z_{1}}{Z_{2}}-\frac{Z_{2}}{Z_{1}}\right)+\left(\frac{Z_{1}}{Z_{2}}+\frac{Z_{2}}{Z_{1}}\right) \cos \left(k_{z 1} f d\right)\right] \\
& \left.\times \sin \left(k_{z 2}[1-f] d\right)\right\}, \\
C= & i Z_{1}\left\{\sin \left(k_{z 1} f d\right) \cos \left(k_{z 2}[1-f] d\right)\right. \\
& -\frac{1}{2}\left[\left(\frac{Z_{1}}{Z_{2}}-\frac{Z_{2}}{Z_{1}}\right)-\left(\frac{Z_{1}}{Z_{2}}+\frac{Z_{2}}{Z_{1}}\right) \cos \left(k_{z 1} f d\right)\right] \\
& \left.\times \sin \left(k_{z 2}[1-f] d\right)\right\} .
\end{aligned}
$$

$Z_{1}$ and $Z_{2}$ are the wave impedances of either TE- or TMpolarized plane waves in dielectric slabs of permittivities $\varepsilon_{1}$ and $\varepsilon_{2}$, respectively, $f$ is the fill fraction of $\varepsilon_{1}, d$ is the thickness of the unit cell, $k_{z 1,2}=\sqrt{\varepsilon_{1,2} k_{0}^{2}-k_{t}^{2}}, k_{t}=k_{0} \sin \theta$, and $\theta$ is the angle of incidence.

\section{APPENDIX C: ANALYSIS OF TOLERANCES AND GROUP DELAY}

We start with tolerances with respect to small changes in the frequency and structure's geometry related to the 1D photonic crystal considered in Sec. III A. Requirements of the design of the unit cells are quite flexible as one does not have to satisfy exact mathematical conditions. However, there is a general rule that a photonic crystal should be formed of alternating high- and low-permittivity dielectric layers, with the fill fraction of the low-permittivity material being small. The influence of the dielectric losses and moderate (5\%) random errors of the layers thicknesses is demonstrated in Fig. 8. The losses do not destroy the effect of zero phase delay but obviously reduce the transmission amplitude at the resonance frequencies, as can clearly be seen from a comparison of Figs. 4(c)-4(e) with Figs. 8(a) and 8(b). The widths and positions of the peaks only slightly change compared with the lossless multilayer. The random errors in addition to the losses affect mainly the phase accumulation, but the latter is still quite small, as shown in Fig. 8(c).

In the main text we deal only with monochromatic plane waves. Generally, a spectral composition of such waves (signal) may propagate with significant distortions due to a complex frequency response of the propagation medium. From Figs. 4(c)-4(e) one can see that the transfer function of the considered 1D photonic crystal (namely, the transmission coefficient $T$ ) significantly depends on the frequency. In order to estimate possible signal distortion due to the nonlinear (with respect to the frequency) phase response we consider group delay $\tau_{g}$, defined as $\partial \operatorname{Arg}[T] / \partial \omega$. Group delay at the frequency $\omega$ can be understood as the time it takes for a signal with a narrow spectrum (centered around $\omega$ ) to traverse the structure [32]. Figure 9 shows the normalized group delay through the 1D photonic crystal considered in the main text as a function of frequency. Results for different polarizations and incidence angles are presented in Figs. 9(a) and 9(b) for a single unit cell. The maxima of the group delay (except the one at $\omega=0$ ) correspond to the top edges of band gaps (in agreement with the well-known slow-light phenomenon [33]), where we expect to observe full transmission without phase delay. Notably, in the case of TE polarization the magnitude of the maxima increases with the incidence angle, while it decreases for TM polarization. Group delay increases, and additional maxima appear when one adds unit cells to the photonic crystal slab [see Fig. 9(c)]. Overall, we can conclude that only signals with small bandwidth can be used for transferring information through such a photonic crystal possessing ENZ properties.
[1] S. Jahani and Z. Jacob, All-dielectric metamaterials, Nat. Nanotechnol. 11, 23 (2016).

[2] S. A. Cummer, J. Christensen, and A. Alù, Controlling sound with acoustic metamaterials, Nat. Rev. Mater. 1, 16001 (2016).

[3] S. B. Glybovski, S. A. Tretyakov, P. A. Belov, Y. S. Kivshar, and C. R. Simovski, Metasurfaces: From microwaves to visible, Phys. Rep. 634, 1 (2016).

[4] K. Sun, R. Fan, X. Zhang, Z. Zhang, Z. Shi, N. Wang, P. Xie, Z. Wang, G. Fan, H. Liu, C. Liu, T. Li, C. Yan, and Z. Guo, An overview of metamaterials and their achievements in wireless power transfer, J. Mater. Chem. C 6, 2925 (2018).
[5] X. C. Tong, Functional Metamaterials and Metadevices (Springer, Cham, Switzerland, 2018).

[6] A. Poddubny, I. Iorsh, P. Belov, and Y. Kivshar, Hyperbolic metamaterials, Nat. Photonics 7, 948 (2013).

[7] V. P. Drachev, V. A. Podolskiy, and A. V. Kildishev, Hyperbolic metamaterials: New physics behind a classical problem, Opt. Express 21, 15048 (2013).

[8] R. Liu, Q. Cheng, T. Hand, J. J. Mock, T. J. Cui, S. A. Cummer, and D. R. Smith, Experimental Demonstration of Electromagnetic Tunneling Through an Epsilon-Near-Zero Metamaterial at Microwave Frequencies, Phys. Rev. Lett. 100, 023903 (2008). 
[9] B. Edwards, A. Alù, M. E. Young, M. Silveirinha, and N. Engheta, Experimental Verification of Epsilon-Near-Zero Metamaterial Coupling and Energy Squeezing Using a Microwave Waveguide, Phys. Rev. Lett. 100, 033903 (2008).

[10] A. Alù, M. G. Silveirinha, A. Salandrino, and N. Engheta, Epsilon-near-zero metamaterials and electromagnetic sources: Tailoring the radiation phase pattern, Phys. Rev. B 75, 155410 (2007).

[11] C. Argyropoulos, G. D'Aguanno, and A. Alù, Giant secondharmonic generation efficiency and ideal phase matching with a double $\epsilon$-near-zero cross-slit metamaterial, Phys. Rev. B 89, 235401 (2014).

[12] M. A. Vincenti, D. de Ceglia, A. Ciattoni, and M. Scalora, Singularity-driven second- and third-harmonic generation at $\epsilon$-near-zero crossing points, Phys. Rev. A 84, 063826 (2011).

[13] C. Argyropoulos, P.-Y. Chen, G. D'Aguanno, N. Engheta, and $\mathrm{A}$. Alù, Boosting optical nonlinearities in $\epsilon$-near-zero plasmonic channels, Phys. Rev. B 85, 045129 (2012).

[14] S. Feng, Loss-Induced Omnidirectional Bending to the Normal in $\epsilon$-Near-Zero Metamaterials, Phys. Rev. Lett. 108, 193904 (2012).

[15] I. Liberal and N. Engheta, Near-zero refractive index photonics, Nat. Photonics 11, 149 (2017).

[16] I. Liberal, A. M. Mahmoud, Y. Li, B. Edwards, and N. Engheta, Photonic doping of epsilon-near-zero media, Science 355, 1058 (2017).

[17] N. Garcia, E. V. Ponizovskaya, and J. Q. Xiao, Zero permittivity materials: Band gaps at the visible, Appl. Phys. Lett. 80, 1120 (2002).

[18] M. H. Javani and M. I. Stockman, Real and Imaginary Properties of Epsilon-Near-Zero Materials, Phys. Rev. Lett. 117, 107404 (2016).

[19] X. Huang, Y. Lai, Z. H. Hang, H. Zheng, and C. T. Chan, Dirac cones induced by accidental degeneracy in photonic crystals and zero-refractive-index materials, Nat. Mater. 10, 582 (2011).

[20] P. Moitra, Y. Yang, Z. Anderson, I. I. Kravchenko, D. P. Briggs, and J. Valentine, Realization of an all-dielectric zero-index optical metamaterial, Nat. Photonics 7, 791 (2013)
[21] N. C. Panoiu, R. M. Osgood, Jr., S. Zhang, and S. R. J. Brueck, Zero- $n$ bandgap in photonic crystal superlattices, J. Opt. Soc. Am. B 23, 506 (2006).

[22] S. Kocaman, M. S. Aras, P. Hsieh, J. F. McMillan, C. G. Biris, N. C. Panoiu, M. B. Yu, D. L. Kwong, A. Stein, and C. W. Wong, Zero phase delay in negative-refractive-index photonic crystal superlattices, Nat. Photonics 5, 499 (2011).

[23] David M Pozar, Microwave Engineering (Wiley, Hoboken, NJ, 2009).

[24] V. Popov, A. V. Lavrinenko, and A. Novitsky, Surface waves on multilayer hyperbolic metamaterials: Operator approach to effective medium approximation, Phys. Rev. B 97, 125428 (2018).

[25] J. M. Bendickson, J. P. Dowling, and M. Scalora, Analytic expressions for the electromagnetic mode density in finite, onedimensional, photonic band-gap structures, Phys. Rev. E 53, 4107 (1996).

[26] A. Figotin and I. Vitebskiy, Electromagnetic unidirectionality in magnetic photonic crystals, Phys. Rev. B 67, 165210 (2003).

[27] A. Figotin and I. Vitebskiy, Gigantic transmission band-edge resonance in periodic stacks of anisotropic layers, Phys. Rev. E 72, 036619 (2005).

[28] Y. Lai, J. Ng, H.-Y. Chen, D.-Z. Han, J.-J. Xiao, Z.-Q. Zhang, and C. T. Chan, Illusion Optics: The Optical Transformation of an Object into Another Object, Phys. Rev. Lett. 102, 253902 (2009).

[29] W. X. Jiang, H. F. Ma, Q. Cheng, and T. J. Cui, Illusion media: Generating virtual objects using realizable metamaterials, Appl. Phys. Lett. 96, 121910 (2010).

[30] P. H. Tichit, S. N. Burokur, J. Yi, and A. de Lustrac, Transformation electromagnetics for antennas with an illusion on the radiation pattern, IEEE Antennas Wireless Propag. Lett. 13, 1796 (2014).

[31] Z. Yao, J. Luo, and Y. Lai, Illusion optics via one-dimensional ultratransparent photonic crystals with shifted spatial dispersions, Opt. Express 25, 30931 (2017).

[32] A. V. Oppenheim, A. S. Willsky, and S. H. Nawab, Signals and Systems, 2nd ed. (Prentice-Hall, Upper Saddle River, NJ, 1996).

[33] J. P. Dowling, M. Scalora, M. J. Bloemer, and C. M. Bowden, The photonic band edge laser: A new approach to gain enhancement, J. Appl. Phys. 75, 1896 (1994). 